\title{
Automatic Design System with Generative Adversarial Network and Convolutional Neural Network for Optimization Design of Interior Permanent Magnet Synchronous Motor
}

\author{
Yuki Shimizu, Student Member, IEEE, Shigeo Morimoto, Member, IEEE, \\ Masayuki Sanada, Member, IEEE, and Yukinori Inoue, Member, IEEE
}

\begin{abstract}
The optimal design of interior permanent magnet synchronous motors requires a long time because finite element analysis (FEA) is performed repeatedly. To solve this problem, many researchers have used artificial intelligence to construct a prediction model that can replace FEA. However, because the training data are generated by FEA, it takes a very long time to obtain a sufficient amount of data, making it impossible to train a large-scale prediction model. Here, we propose a method for generating a large amount of data from a small number of FEA results using machine learning. An automatic design system with a deep generative model and a convolutional neural network is then constructed. With its sufficient data, the proposed system can handle three topologies and three motor parameters in a wide range of current vector regions. The proposed system was applied to multi-objective optimization design, with the optimization completed in 13-15 seconds.
\end{abstract}

Index Terms-Convolutional neural network, design optimization, generative adversarial network, permanent magnet motor, semisupervised learning.

\section{INTRODUCTION}

$I^{1}$ NTERIOR permanent magnet synchronous motors (IPMSMs) are widely adopted in electric vehicles and industrial robots because of their high output, efficiency, and reliability [1], [2]. However, a major problem with IPMSMs is their long optimization period, which is caused by the high degree of freedom in their design and the use of finite element analysis (FEA).

Regarding the high degree of freedom in design, an IPMSM rotor can be designed with a large number of geometries due to the embedded permanent magnet (PM). Various design alternatives thus need to be considered during optimization. Sun et al. [3] classified the dimensions of IPMSMs into three categories based on a factor analysis and performed multi-objective optimization. Islam et al. [4] optimized two rotor dimensions at multiple output points of an IPMSM using the response surface method. These dimensional optimizations were effective for optimizing the shape for a given topology. However, it is difficult to deal with multiple topologies because the geometries depend on the initial shape. To solve this problem, many studies have proposed rotor design based on topology optimization. Ishikawa et al. [5] minimized PM volume using multi-material topology optimization for an asymmetric IPMSM rotor. Sato et al. [6] applied multi-material topology optimization to an IPMSM rotor using a normalized Gaussian network. Although these methods produce completely new topologies, some topologies cannot be manufactured and a very large number of candidate solutions must be considered in the optimization due to the huge design space. There is thus a need for a method for generating manufacturable design alternatives with multiple topologies in a small design variable space.

Regarding electromagnetic modeling, finite element analysis (FEA), a numerical modeling method, is generally used to calculate the characteristics of an IPMSM. FEA can be used to obtain very accurate operating characteristics of IPMSMs, but it is time-consuming. Many researchers have thus investigated the construction of prediction models using artificial intelligence (AI) to reduce analysis time while maintaining the accuracy of FEA. Dhulipati et al. [7] used support vector regression (SVR) to train a prediction model for a six-phase IPMSM. Hao et al. [8] trained a model to learn the relationship between the dimensions and torque ripple of an IPMSM using radial basis function networks, and used the model for optimization. Pan et al. [9] used XGBoost to learn the relationship between the torque characteristics and the structural parameters of permanent magnet arc motors, and used the model for optimization. Barmada et al. [10] used a convolutional neural network (CNN) to learn the relationship between the shape and torque characteristics of synchronous reluctance motors, and used the $\mathrm{CNN}$ for optimization. Asanuma et al. [11] trained a model to learn the relationship between the topology near the rotor surface and the torque characteristics of an IPMSM using transfer learning and used the model for optimization. These conventional AI-based modeling methods achieve high accuracy but take into account only specific geometries or one current vector condition, making them unsuitable for various applications. Therefore, there is a need for a more general modeling technique.

The small scale of the above AI-based modeling techniques 
is due to the difficulty in obtaining sufficient data. For example, when training a prediction model to replace FEA, the training data are generally generated by FEA. Assuming that the FEA for generating training data takes 12.4 minutes to analyze the speed-torque characteristics of an IPMSM [12], a FEA of 100,000 datasets would take more than two years.

This study solves the problem of obtaining sufficient data by applying deep learning models to design and modeling. Fig. 1 shows an overview of the present study. First, for data acquisition, we use semi-supervised learning where the training data are generated using machine learning. In [12], the authors proposed a method for training a prediction model that can accurately predict the speed-torque characteristics of double-layered IPMSMs from a small number of FEA results using machine learning. This prediction model can be used to calculate the operating characteristics of various double-layered IPMSMs from their rotor shape to generate a large dataset in a short time. Thus, by constructing a machine learning model that is limited to a certain typical rotor shape from a small dataset, and applying the data generation process to various shapes, we can quickly obtain sufficient data.

Using the generated data, an automatic rotor core design system based on two deep learning models is constructed. The design system consists of a deep generative model to design the rotor core and a characteristic prediction model to predict the characteristics of the generated rotor design. The deep generative model uses a generative adversarial network (GAN) and the characteristic prediction model uses a CNN. The two trained deep learning models are then used for multi-objective optimization design. It is shown that the design time is significantly reduced. The contributions of this paper can be summarized as follows.

a) A quick training data generation method for large-scale deep learning is proposed.

b) A deep generative model that integrally represents different topologies in the latent space is applied.

c) A prediction model of FEA that can be applied in a wide range of current vector regions is presented.

d) An automatic design system for IPMSMs that enables quick design is proposed.

The rest of this paper is organized as follows. Section II describes the proposed method for quickly generating training data. Section III describes deep learning based on the generated dataset. Section IV shows the results of torque maximization and PM volume minimization using the proposed automatic design system. Section V summarizes the results.

\section{OBtAining TRAINING DATA}

Semi-supervised learning with machine learning is used to quickly generate a sufficient amount of data for training the deep learning models. In this section, we describe the process of generating training data.

The target of this study is an IPMSM with 8 poles and 48 slots of distributed winding stators. Fig. 2 shows the rotor topologies of 2D-, V-, and Nabla-structure IPMSMs [2], [13].

In this study, we generate data based on these three types of topology. The magnetic materials are the same as those in [14],

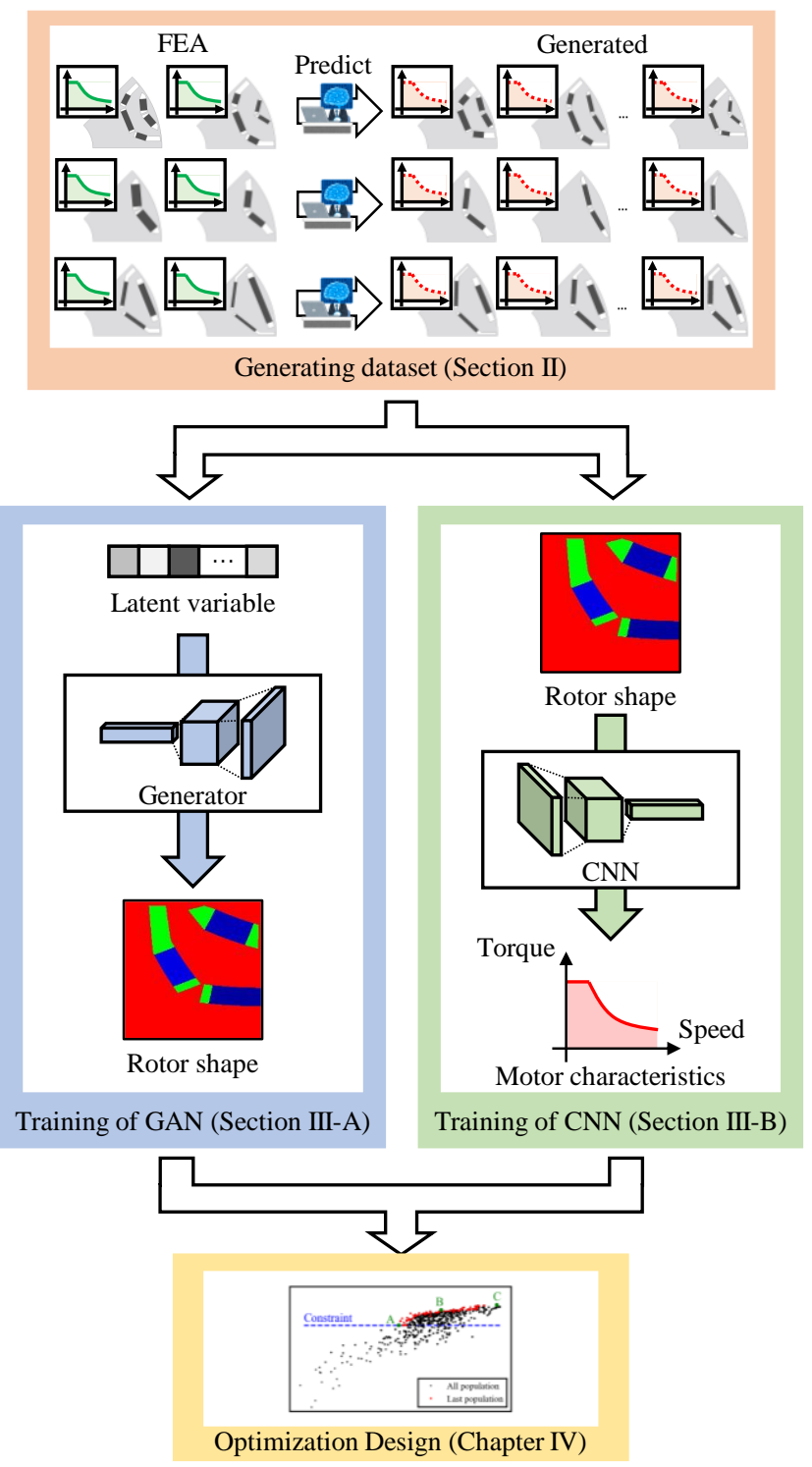

Fig. 1. Overview of this study.

i.e., the magnet is NMX-S49CH and the iron core is 10JNEX900. See [2] for detailed information on the stator geometry, body size, and other parameters.

First, the relationship between rotor shape and speed-torque characteristics is learned using the method proposed in [12]. For the prediction model in [12], the input variables are the geometry and current conditions. The geometry is represented numerically using dimensional information, such as the PM thickness, as parameters. Although the objective is to predict the speed-torque characteristics, the prediction models do not directly predict the speed-torque characteristics; instead, we set three motor parameters (PM flux linkage and $d$ - and $q$-axis inductances) as the prediction targets to improve accuracy and calculate the speed-torque characteristics. Table I shows the machine learning methods used to predict the characteristics for each geometry, where GPR is Gaussian process regression. The values in parentheses are the coefficients of determination for the test data. For the training data, we used a total of 26,209 randomly generated shapes (2D: 7,927; V: 8,256; Nabla: 
(a)

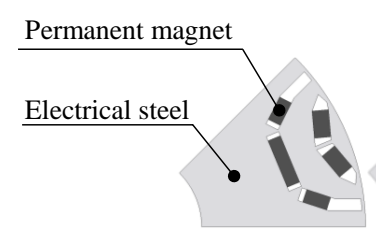

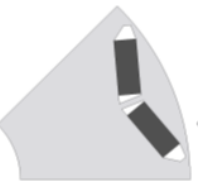

(b)

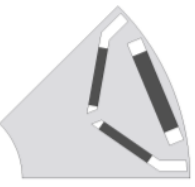

(c)
Fig. 2. Single-pole conventional rotor shapes. (a) 2D, (b) V, and (c) Nabla.

10,026), all of which were analyzed under random current vector conditions. The software JMAG-Designer 19.1 was used for the FEA. The effects of the imbalance in prediction accuracy for each topology and each parameter are described in Section IV.

The trained prediction models were then used to generate shape and motor parameter pairs for 55,000 shapes for each topology, for a total of 165,000 shapes. Because the motor parameters change nonlinearly due to the effect of magnetic saturation, we predicted the change in motor parameters versus the current vector condition for each shape. Because the maximum armature current of the IPMSM used in this research is $232 \mathrm{~A}$, the characteristic data generated by the prediction are the discrete PM flux linkage with an armature current ranging from 5 to $235 \mathrm{~A}$ in 5-A increments and the discrete $d$ - and $q$-axis current vs. $d$ - and $q$-axis inductance characteristics with $d$ - and $q$-axis currents ranging from 5 to $235 \mathrm{~A}$ in $10-\mathrm{A}$ increments (see Fig. 5). Here, the PM flux linkage is assumed to be independent of the current phase [12].

The data augmentation method described above predicted the characteristics for 623 current vector conditions for each shape, meaning that the FEA results for 102,795,000 conditions $(165,000$ shapes $\times 623$ conditions/shape) were predicted from FEA results for only 24,000 conditions. The generation (prediction) of 102,795,000 data points was completed in a total of 3.6 hours. We were thus able to obtain sufficient data in a practical amount of time.

\section{Automatic Design System}

Using the dataset created in Section II, we trained the deep learning models for the automatic rotor shape design system. The automatic design system consists of two types of deep learning model, one for design and the other for characteristic prediction. This section describes the training methods and the results for these two deep learning models.

\section{A. Training of Generative Adversarial Network}

The first deep learning model is a deep generative model for designing rotor shapes.

First, we describe the numerical representation of the motor shape. There are two types of numerical representation of motor shapes, namely that used in [12] (see Section II) that specifies the dimensions of the shape, denoted as the dimensional representation, and that used in topology optimization that specifies the material at each coordinate, denoted as the material representation. The dimensional representation can represent only one topology depending on the reference shape and is unsuitable for a system that handles multiple topologies in an integrated manner. For example, the
TABLE I

\begin{tabular}{cccc} 
MACHINE LEARNING METHODS USED FOR DATA GENERATION \\
\hline \multirow{2}{*}{ Topology } & $\Psi_{a}$ & $L_{d}$ & $L_{q}$ \\
\hline \multirow{2}{*}{$2 \mathrm{D}$} & $\mathrm{SVR}$ & $\mathrm{SVR}$ & XGBoost \\
& $(0.997)$ & $(0.963)$ & $(0.998)$ \\
\hline \multirow{2}{*}{$\mathrm{V}$} & $\mathrm{SVR}$ & $\mathrm{SVR}$ & $\mathrm{SVR}$ \\
& $(1.000)$ & $(0.979)$ & $(0.999)$ \\
\hline \multirow{2}{*}{ Nabla } & GPR & SVR & XGBoost \\
& $(0.869)$ & $(0.725)$ & $(0.986)$ \\
\hline \hline
\end{tabular}

geometric parameters used for the 2D, V, and Nabla structures are 11-, 6-, and 8-dimensional, respectively, making it difficult to handle different topologies with a given dimensional representation. Therefore, in the proposed automatic design system, the motor shape is represented numerically by the material representation.

Fig. 3 shows the material representation method used in the proposed system. An electromagnetic steel sheet, a PM, or air is specified for each pole coordinate of the rotor, and the three materials are assigned to the RGB (red, green, blue) values of the image as one-hot vectors, respectively, to represent the rotor shape in the image, as shown in the right image in Fig. 3. Because the shape considered in this study is $d$-axis symmetric and there is no magnet or air layer near the shaft, only half of the geometry in the circumferential direction and $60 \%$ of the geometry in the radial direction are converted into images. The magnetization direction (angle) of the PM is represented by the difference in the brightness of the blue color by inputting the normalized value of the angle information $d_{P M}$, which is calculated as follows:

$$
d_{P M}=\frac{\theta_{P M}+90}{180},
$$

where $\theta_{P M} \in\left[-90^{\circ}, 90^{\circ}\right]$ is the angle of the magnetization direction of each PM. In the actual image generation process for the material representation, the material information at each coordinate was extracted and encoded into an image for the rotor shape whose dimensions were parametrically generated in JMAG-Designer. Note that due to the coordinate transformation, the straight part of the rotor shape becomes a gentle curve in the image.

Material representation has the following two problems.

a) The design space becomes huge.

b) The design space includes shapes that cannot be manufactured.

For example, if a shape is divided into $256 \times 256$ parts and the material is specified, $3^{256 \times 256}$ shapes can be represented, some of which cannot be manufactured (i.e., they are noise). Therefore, when using material representation, it is necessary to extract only the appropriate design space.

In this study, we use a GAN to solve this problem. A GAN is a deep generative model that uses two deep neural networks proposed by Goodfellow et al. [15]. In this study, lightweight GAN [16] is used to learn the 165,000 shapes generated in Section II. The image was a $256 \times 256 \times 3$ tensor and the latent variable was a 256 -dimensional vector. The specific GAN 


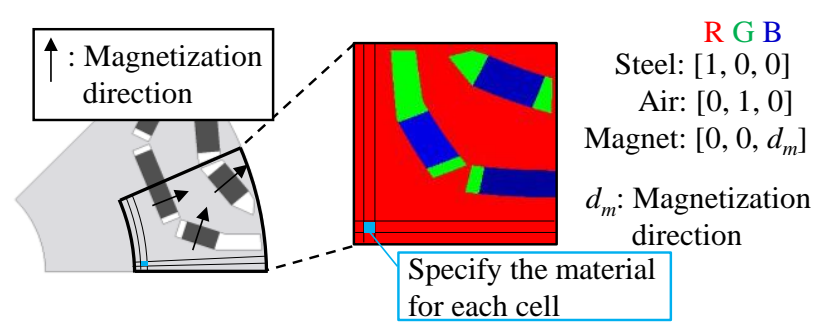

Fig. 3. Material representation of rotor shape.

learning objective in this study is to learn three types of rotor topology, namely 2D, V, and Nabla structures, and to integrally represent them in the latent variable space.

Fig. 4 shows an example of a shape generated by lightweight GAN. The output images of the GAN clearly show the three types of rotor shape. All of these images were sampled from the same latent variable space, indicating that the use of GAN allows us to handle a wide variety of shapes in a unified manner. In addition, the huge design space can be reduced to 256 dimensions of the latent variable space and undesignable shapes can be eliminated, which overcomes the two problems of material representation.

\section{B. Training of Convolutional Neural Network}

If we can evaluate the operating characteristics of the shape designed by the GAN trained in Section III-A, it will be possible to perform a unified optimization design of various shapes by searching the latent variable space of the generative model. A CNN is used instead of the machine learning method used for data generation in Section II for the following reasons:

a) The change from a dimensional representation to a material representation (image) requires the extraction of shape features by a convolutional layer of the CNN for highly accurate characteristic prediction.

b) Conventional SVR and GPR cannot handle big data due to computational cost.

Fig. 5 shows the architecture of the CNN used in this study. The regression CNN is built based on ResNet-18 [17]. It is a multi-task learning architecture that simultaneously predicts three motor parameters for a single shape. Because each motor parameter is nonlinear with respect to the current vector, the characteristic data generated in Section II were approximated by polynomial equations using the least-squares method. The coefficients of the approximation equation were used as the prediction target. The approximation equations are shown below.

$$
\begin{aligned}
\Psi_{a}= & w_{0}^{\Psi_{a}}+w_{1}^{\Psi_{a}} I_{a}+w_{2}^{\Psi_{a}} I_{a}^{2}+w_{3}^{\Psi_{a}} I_{a}^{3}, \\
L_{d}= & w_{0}^{L_{d}}+w_{1}^{L_{d}} i_{d}+w_{2}^{L_{d}} i_{q}+w_{3}^{L_{d}} i_{d}^{2}+w_{4}^{L_{d}} i_{d} i_{q}+w_{5}^{L_{d}} i_{q}^{2} \\
& +w_{6}^{L_{d}} i_{d}^{3}+w_{7}^{L_{d}} i_{d}^{2} i_{q}+w_{8}^{L_{d}} i_{d} i_{q}^{2}+w_{9}^{L_{d}} i_{q}^{3}, \\
L_{q}= & w_{0}^{L_{q}}+w_{1}^{L_{q}} i_{d}+w_{2}^{L_{q}} i_{q}+w_{3}^{L_{q}} i_{d}^{2}+w_{4}^{L_{q}} i_{d} i_{q}+w_{5}^{L_{q}} i_{q}^{2} \\
& +w_{6}^{L_{q}} i_{d}^{3}+w_{7}^{L_{q}} i_{d}^{2} i_{q}+w_{8}^{L_{q}} i_{d} i_{q}^{2}+w_{9}^{L_{q}} i_{q}^{3} \\
& +w_{10}^{L_{q}} i_{d}^{4}+w_{11}^{L_{q}} i_{d}^{3} i_{q}+w_{12}^{L_{q}} i_{d}^{2} i_{q}^{2}+w_{13}^{L_{q}} i_{d} i_{q}^{3}+w_{14}^{L_{q}} i_{q}^{4},
\end{aligned}
$$

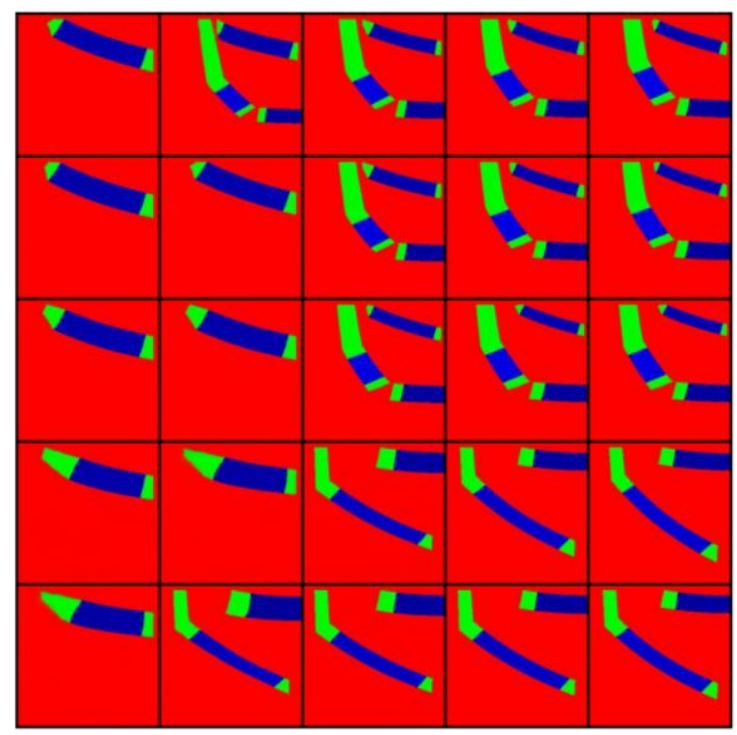

Fig. 4. Example of results generated by lightweight GAN.

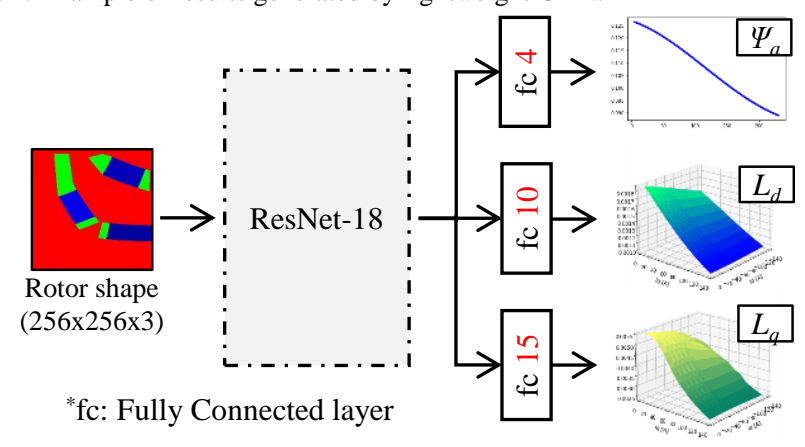

Fig. 5. Architecture of multi-task CNN

where $I_{a}$ is the armature current, $i_{d}$ and $i_{q}$ are the $d$ - and $q$-axis currents, respectively, and $w_{*}^{*}$ is the coefficient. The order of the polynomial approximation was determined by calculating the coefficient of determination for the data predicted by each approximation equation, so that all coefficients of determination for 10 randomly sampled shapes were higher than 0.9995. As shown in Fig. 5, the number of output nodes in fully connected layers was determined according to the number of coefficients in (2)-(4).

From the normalized dataset of 165,000 shapes (combinations of shapes and approximation equation coefficients), 120,000 were used as training data, 30,000 were used as validation data, and 15,000 were used as test data for training. Adam was used for optimization, the weight decay was 0.0001 , and the mini-batch size was 16 . The loss function $\mathcal{L}_{C N N}$ is defined as follows.

$$
\mathcal{L}_{C N N}=\sum_{\text {param }} k^{\text {param }} \operatorname{MSE}\left(\hat{\mathbf{w}}^{\text {param }}, \mathbf{w}^{\text {param }}\right),
$$

where $k^{\text {param }}$ is the coefficient for balancing losses, MSE is a function that returns the mean squared error, $\mathbf{w}^{\text {param }}$ is the true value of the weight vector for each parameter, and $\hat{\mathbf{w}}^{\text {param }}$ is the CNN prediction of $\mathbf{w}^{\text {param }}$. In this study, we set the coefficient $\left(k^{\Psi_{a}}, k^{L_{d}}, k^{L_{q}}\right)=(3,2,1)$.

Fig. 6 shows the prediction accuracy of the trained multi-task 

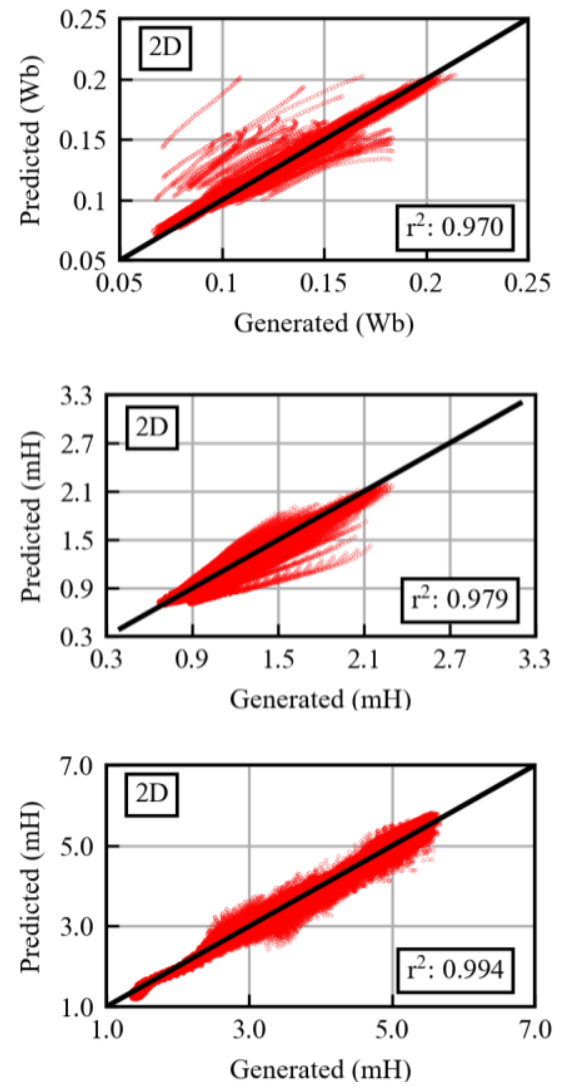

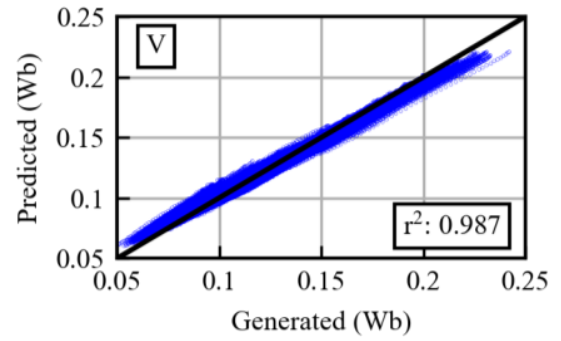

(a)

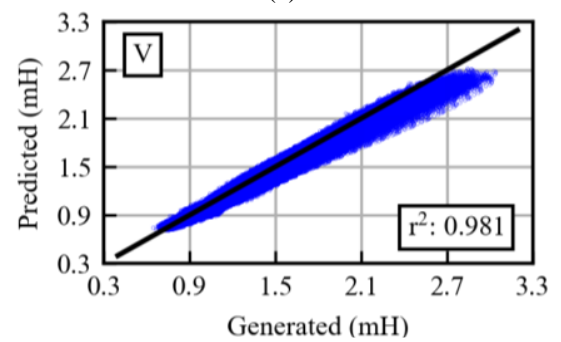

(b)

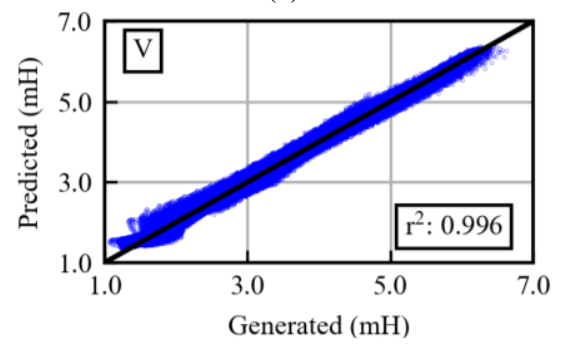

(c)
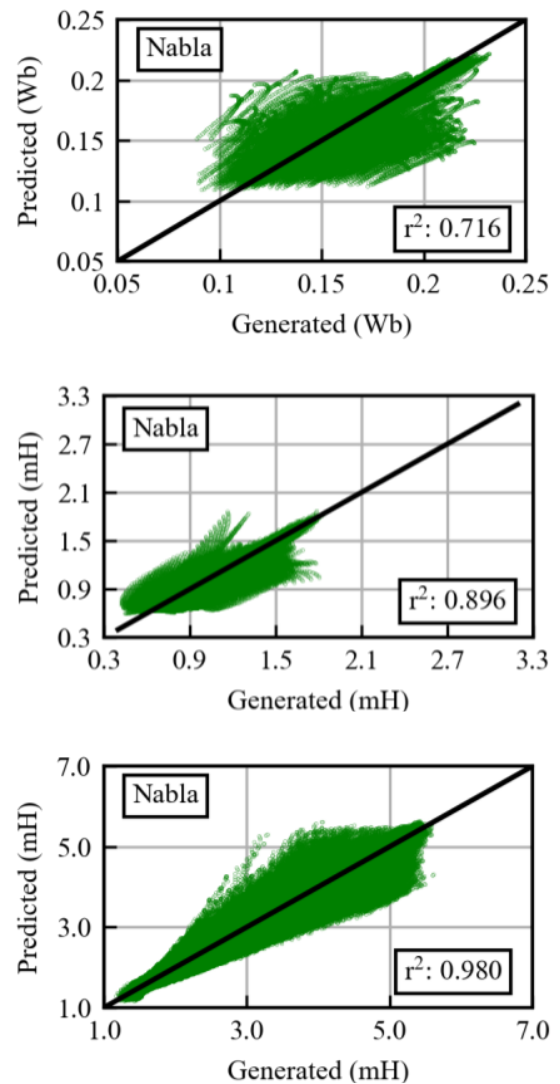

Fig. 6. Prediction results for test data, where $\mathrm{r}^{2}$ is the coefficient of determination. Results for (a) PM flux linkage, (b) $d$-axis inductance, (c) and $q$-axis inductance.

CNN on the test data. Note that this prediction accuracy is not for the FEA results, but for the data generated by machine learning. A comparison of the prediction results indicates that the accuracy was high except for the PM flux linkage and $d$-axis inductance in the Nabla structure. The prediction accuracy of these two characteristics by machine learning in the data generation stage shown in Table I is also low, which may be due to the prediction error generated during data generation. Because errors are included in the generated data (the target of prediction), the low prediction accuracy does not imply that the prediction accuracy for the FEA characteristics is also low. The prediction accuracy for FEA is discussed in Section IV.

\section{Optimization DESIGN}

The combination of the deep generative model and the characteristic prediction model in Section III results in the architecture shown in Fig. 7. This section demonstrates the usefulness of the system by performing a multi-objective optimization design in the latent variable space of the generative model.

\section{A. Problem}

In this study, the design goals are to minimize the volume of the PMs and maximize the maximum torque under the torque constraint. The problem setup is as follows:

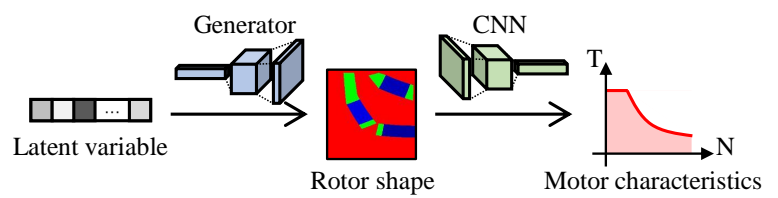

Fig. 7. Overall design system.

$$
\begin{aligned}
& \min \quad w_{1} \frac{V_{P M}}{V_{\text {init }}}-w_{2} \frac{T_{\text {pred }}^{\text {Max }}}{T_{\text {init }}^{\text {Max }}}, \\
& \text { s.t. } \quad g_{i}: T_{\text {pred }}^{(i)} \geq \alpha T_{\text {req }}^{(i)}(i=1,2, \ldots, p),
\end{aligned}
$$

where $V_{P M}$ is the volume of a PM for each candidate solution and $T_{\text {pred }}$ is the predicted maximum torque for each candidate solution. These parameters are normalized by the initial values $V_{\text {init }}$ and $T_{\text {init }}^{M a x}$, respectively. The volume of the PM was defined as a percentage of the image area. $w_{1}$ and $w_{2}$ are weight coefficients. $\left(w_{1}, w_{2}\right)=(1,1)$ in this setup. The constraint condition $g_{i}$ is a torque constraint for $p$ required operating points $\left\{\left(N_{\text {req }}^{(i)}, T_{\text {req }}^{(i)}\right)\right\}_{i=1}^{p}$, which is multiplied by a coefficient $\alpha=$ 1.03 to consider the prediction error. The torque prediction results are given as the results of maximum output control at the required speed as follows:

$$
\begin{aligned}
T_{\text {pred }}^{(i)}=\max _{I_{a} \in\left(0, I_{a m}\right], \beta \in(0,90)} & T_{C N N}\left(I_{a}, \beta\right), \\
\text { s.t. } \quad & N_{C N N}\left(I_{a}, \beta\right) \geq N_{\text {req }}^{(i)},
\end{aligned}
$$

where $I_{a m}$ is the maximum armature current, and $T_{C N N}$ and $N_{C N N}$ are the torque and limit speed predicted by the CNN, 


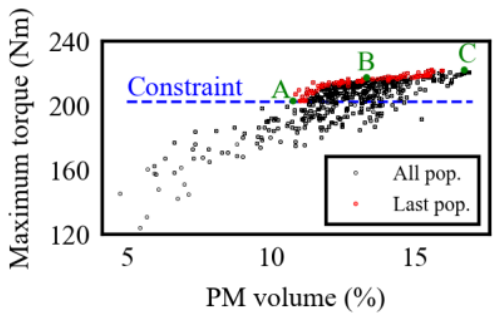

(a)

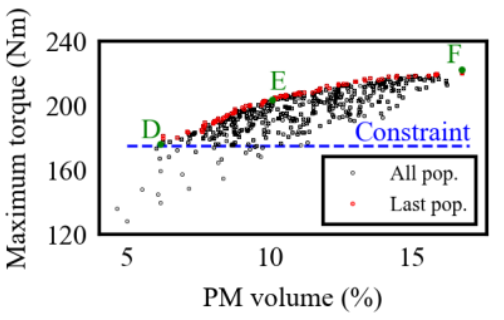

(b)

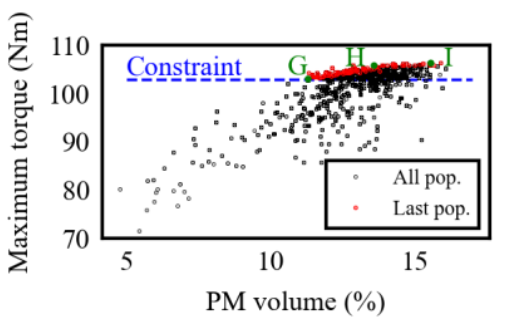

(c)

Fig. 8. Characteristics of all populations and the last population for optimization design under (a) Condition 1 , (b) Condition 2 , (c) and Condition 3.

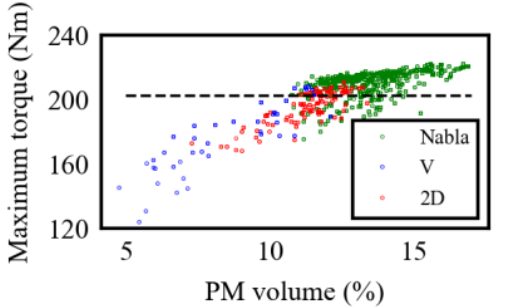

(a)

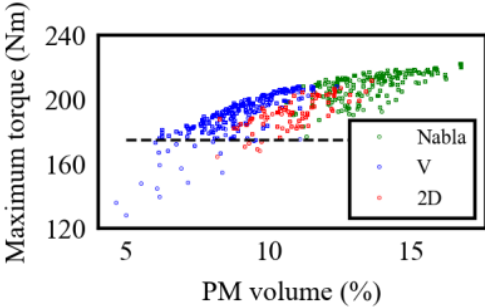

(b)

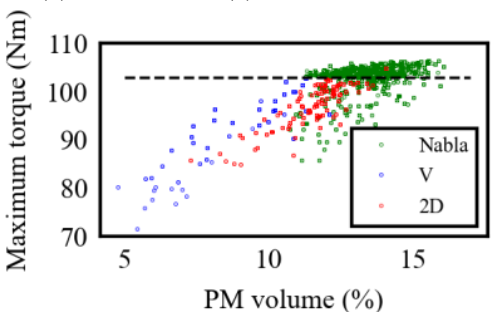

(c)

Fig. 9. Characteristics of all populations with topology information for optimization design under (a) Condition 1 , (b) Condition 2 , and (c) Condition 3.

respectively. The solution for maximum power control was obtained by a brute-force search.

NSGA-II [18] was used as the optimization algorithm, and the framework pymoo was used for the implementation [19]. The population size was set to 100 and the number of offspring was set to 10. Latin hypercube sampling was used for sampling the initial population, the tournament method was used for selection, simulated binary crossover was used for crossover, and polynomial mutation was used for mutation. The termination condition was set to 100 generations.

\section{B. Optimization Results}

To verify the robustness of the proposed system, this study performed optimization under three conditions. Table II shows the settings for the three conditions and Figs. 8 and 9 show the characteristics of all the populations generated in the optimization process under each condition. The maximum armature voltage was set to $507 \mathrm{~V}$.

In Condition 1, the maximum armature current was set to $232 \mathrm{~A}$, and the optimization was performed at two required operating points, $\left(3,000 \mathrm{~min}^{-1}, 197 \mathrm{Nm}\right)$ and $\left(11,000 \mathrm{~min}^{-1}, 40\right.$ $\mathrm{Nm}$ ), which were determined based on the reference motor in [2]. The solution population transitioned to satisfy the constraints; all the individuals in the final population satisfied the constraints. The Nabla structure most easily produced the maximum torque. Almost all of the Pareto solutions are the Nabla structure under severe torque requirements.

In Condition 2, the maximum armature current was fixed at $232 \mathrm{~A}$ and the torque constraint was relaxed from that in Condition 1. Optimization was performed at two required operating points, $\left(3,000 \mathrm{~min}^{-1}, 170 \mathrm{Nm}\right)$ and $\left(11,000 \mathrm{~min}^{-1}, 40\right.$ $\mathrm{Nm})$. In Condition 2, many candidate solutions for all three topologies satisfy the relaxed torque constraint. The $\mathrm{V}$ structure reduced the volume of PMs the most while maintaining high torque. The 2D structure, which was designed for high efficiency [2], does not appear in the Pareto solution for Condition 2.
TABLE II

OPTIMIZATION CONDITIONS

\begin{tabular}{ccc}
\hline $\begin{array}{c}\text { Condition } \\
\text { No. }\end{array}$ & Required operating points & $\begin{array}{c}\text { Maximum } \\
\text { armature current } I_{a m}\end{array}$ \\
\hline Condition 1 & $\begin{array}{c}\left(3,000 \mathrm{~min}^{-1}, 197 \mathrm{Nm}\right) \\
\left(11,000 \mathrm{~min}^{-1}, 40 \mathrm{Nm}\right)\end{array}$ & $232 \mathrm{~A}$ \\
\hline Condition 2 & $\begin{array}{c}\left(1,000 \mathrm{~min}^{-1}, 170 \mathrm{Nm}\right) \\
\left(11,000 \mathrm{~min}^{-1}, 40 \mathrm{Nm}\right)\end{array}$ & $232 \mathrm{~A}$ \\
\hline Condition 3 & $\begin{array}{c}\left(1,000 \mathrm{~min}^{-1}, 100 \mathrm{Nm}\right) \\
\left(9,000 \mathrm{~min}^{-1}, 30 \mathrm{Nm}\right)\end{array}$ & $104 \mathrm{~A}$ \\
\hline \hline
\end{tabular}

In Condition 3, the armature current limit was reduced from 232 to $104 \mathrm{~A}$ and the two required operating points were set to $\left(1000 \mathrm{~min}^{-1}, 100 \mathrm{Nm}\right)$ and $\left(9000 \mathrm{~min}^{-1}, 30 \mathrm{Nm}\right)$. In this condition, the maximum torque requirement was reduced, but the current limit was also reduced, resulting in a tighter torque requirement. In addition, as in Condition 1, many Nabla structures were selected to easily obtain the torque. Thus, the optimization design of the proposed system can be performed under various current limits.

To verify the validity of the optimized design, FEA needs to be performed. To perform FEA, it is necessary to decode the image data for the material representation into computer-aided design (CAD) data. The image generated by the GAN is basically one of the three learned topologies, so it is only necessary to extract the dimensional information (e.g., PM thickness) from the image. The dimension information is obtained using rule-based image recognition. Fig. 10 shows an example of the decoding method for extracting the dimensional information for the first-layer PM of the 2D structure. First, the shape image is converted from RGB to HSV (hue, saturation, value). Only blue cells are extracted from the hue information and converted to binary images of PM locations (value of 1); other cells are set to 0 . Then, we detect the region formed by adjacent non-zero pixels (PM region) as an object and calculate its contour and center of gravity. Based on the center of gravity of the object, the object closest to the rotor surface is determined to be the first-layer PM, and the coordinates of its 


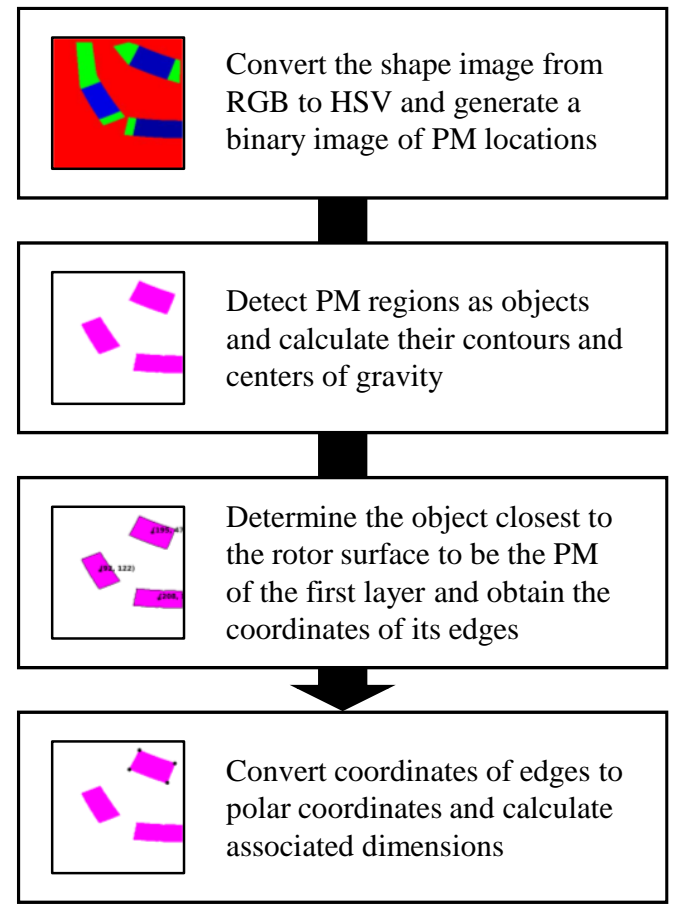

Fig. 10. Example of decoding method for extracting dimensional information.

edge are obtained. By converting these coordinates to polar coordinates, the position of the PM and its associated dimensions can be calculated. By carrying out the above process for the appropriate object in the image, it is possible to decode the shape image into CAD data. In the actual decoding process, we further encode the decoded CAD data and calculate the reconstruction error rate (the percentage of pixel information that does not match). If the reconstruction error rate is over $1 \%$, we re-dimension the $\mathrm{CAD}$ data until the rate becomes less than $1 \%$.

Using the decoding method described above, FEA was conducted by selecting three candidate solutions for each condition from the Pareto solutions. Fig. 11 shows the prediction results produced by the system and the FEA results for the speed-torque characteristics of the selected candidate solutions, where solutions A-I correspond to the solutions in Fig. 8 and the blue points represent the required operating points for each condition.

The shapes (images) of the Pareto solutions are all clear and designable, confirming the effectiveness of the trained GAN. A comparison of the FEA results with the CNN prediction results indicates that the prediction accuracy of the speed-torque characteristics is very high for all candidate solutions. The FEA results for all Pareto solutions satisfy the required operating point, which means that the prediction error is less than $3 \%$. Considering these results, we can conclude that the poor prediction accuracy for the Nabla structure mentioned in Section II was improved by the CNN. In other words, the CNN recognized and eliminated as noise the insensitive error and standard error generated in the process of data generation by SVR and GPR, respectively. This shows that the proposed data generation method can tolerate a certain degree of inaccuracy in a machine learning method.
Finally, we discuss the optimization time. Fig. 12 shows a histogram of the design time for 100 optimization designs under each condition. For the calculations, a computer with an Intel Core $^{\mathrm{TM}} \mathrm{i7}-9700 \mathrm{~K}$ CPU, $32.0 \mathrm{~GB}$ of RAM, and an NVIDIA GeForce RTX 2070 SUPER (8 GB) GPU was used. The proposed system can design a shape that satisfies the requirements in 13-15 seconds, effectively reducing the optimization time compared to that for the conventional optimization calculation for the same scale (several days to several weeks).

\section{CONCLUSION}

This paper proposed a deep learning technique for optimizing IPMSM rotors. The results can be summarized as follows.

a) We proposed a method for quickly generating a large amount of FEA data for training large-scale deep learning models using machine learning specific to each topology. This method generated 102,795,000 training data from 26,209 FEA results.

b) The proposed generative model can be used to design rotor geometries for three IPMSMs with high precision and can represent different topologies in a unified 256-dimensional latent variable space.

c) We proposed a prediction model that can quickly and accurately predict motor parameters in a wide range of current vectors with various geometries.

d) We proposed an automatic rotor design system for IPMSMs using two deep learning models. The proposed system can be used for various required operating points and current ranges. The time required for optimal design was only 13-15 seconds.

The dataset generated in Section II is available at IEEE DataPort [20] and the Python implementation of the deep learning models is available at GitHub [21].

\section{REFERENCES}

[1] Y.-H. Jung, M.-R. Park, K.-O. Kim, J.-W. Chin, J.-P. Hong, and M.-S. Lim, "Design of High-Speed Multilayer IPMSM Using Ferrite PM for EV Traction Considering Mechanical and Electrical Characteristics," IEEE Trans. Ind. Appl., vol. 57, no. 1, pp. 327-339, Jan. 2021.

[2] Y. Shimizu, S. Morimoto, M. Sanada, and Y. Inoue, "Influence of Permanent Magnet Properties and Arrangement on Performance of IPMSMs for Automotive Applications," IEEJ Journal of Industry Applications, vol. 6, no. 6, pp. 401-408, 2017.

[3] X. Sun, Z. Shi, G. Lei, Y. Guo, and J. Zhu, "Multi-Objective Design Optimization of an IPMSM Based on Multilevel Strategy," IEEE Trans. Ind. Electron., vol. 68, no. 1, pp. 139-148, Jan. 2021.

[4] M. S. Islam, M. Chowdhury, A. Shrestha, M. Islam, and I. Husain, "Multiload Point Optimization of Interior Permanent Magnet Synchronous Machines for High-Performance Variable-Speed Drives," IEEE Trans. Ind. Appl., vol. 57, no. 1, pp. 427-436, Jan. 2021.

[5] T. Ishikawa, S. Mizuno, and N. Krita, "Topology Optimization Method for Asymmetrical Rotor Using Cluster and Cleaning Procedure," IEEE Trans. Magn., vol. 53, no. 6, pp. 1-4, Jun. 2017.

[6] T. Sato, K. Watanabe, and H. Igarashi, "Multimaterial Topology Optimization of Electric Machines Based on Normalized Gaussian Network," IEEE Trans. Magn., vol. 51, no. 3, pp. 1-4, Mar. 2015.

[7] H. Dhulipati, E. Ghosh, S. Mukundan, P. Korta, J. Tjong, and N. C. Kar, "Advanced Design Optimization Technique for Torque Profile Improvement in Six-Phase PMSM Using Supervised Machine Learning for Direct-Drive EV," IEEE Trans. Energy Convers., vol. 34, no. 4, pp. 2041-2051, Dec. 2019 


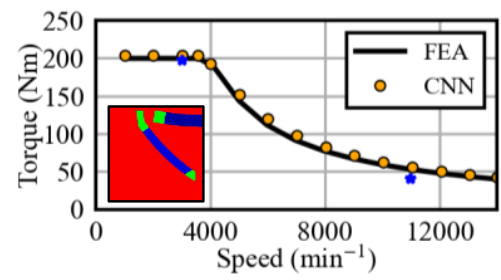

(a)

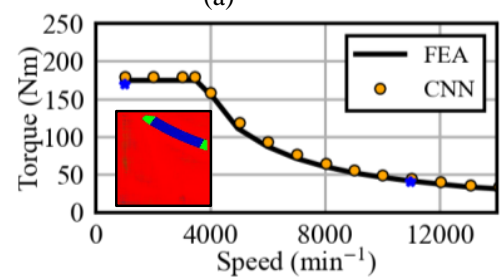

(d)

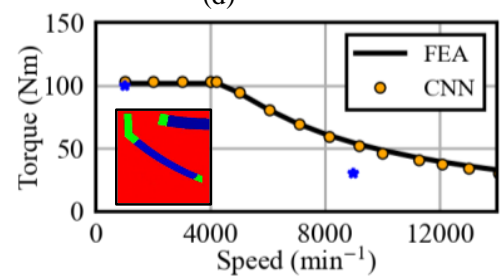

(g)

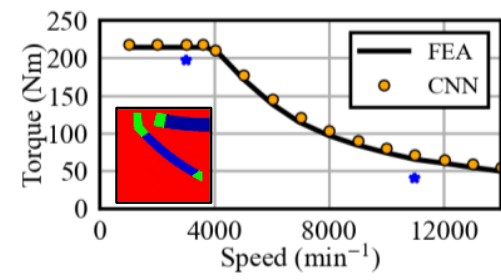

(b)

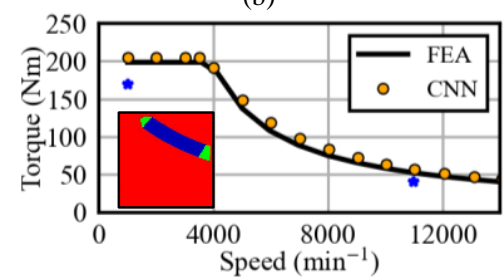

(e)

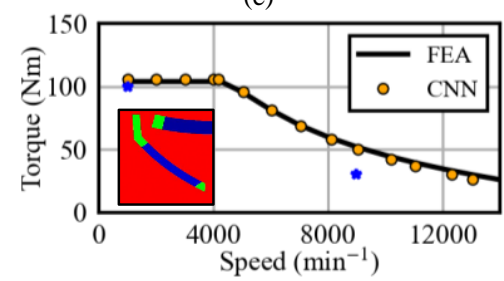

(h)

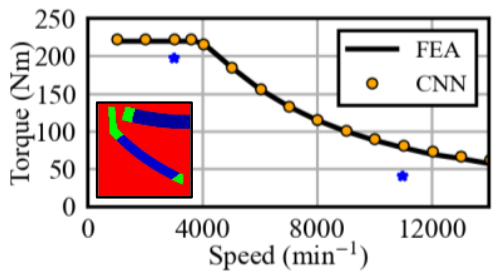

(c)

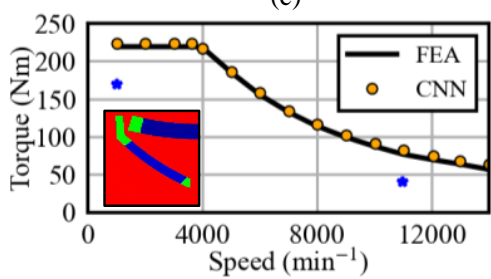

(f)

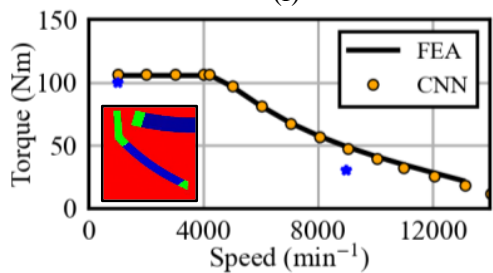

(i)

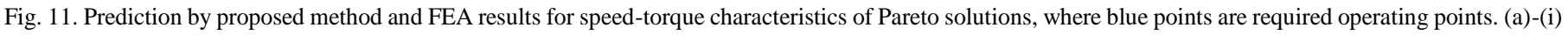
Solutions A-I defined in Fig. 8..

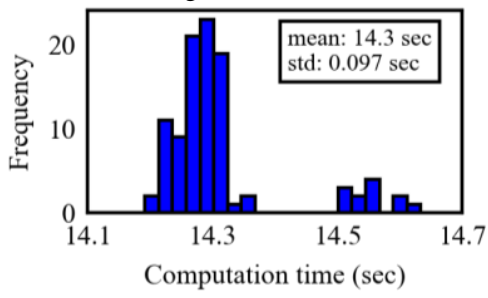

(a)

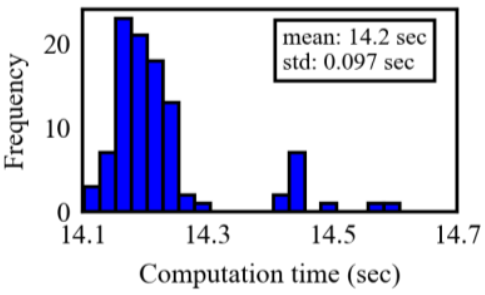

(b)

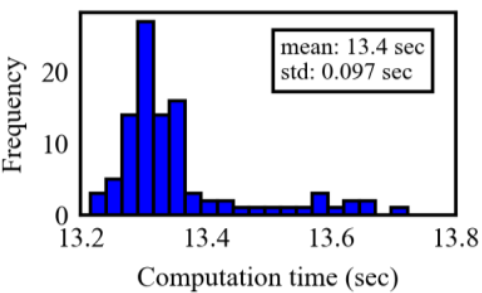

(c)

Fig. 12. Optimization time for (a) Condition 1, (b) Condition 2, and (c) Condition 3 (std: standard deviation).

[8] J. Hao, S. Suo, Y. Yang, Y. Wang, W. Wang, and X. Chen, "Optimization of Torque Ripples in an Interior Permanent Magnet Synchronous Motor Based on the Orthogonal Experimental Method and MIGA and RBF Neural Networks," IEEE Access, vol. 8. pp. 27202-27209, 2020.

[9] Z. Yao, J. Zhao, J. Song, F. Dong, Z. He, and K. Zong, "Research on Selection Criterion of Design Tolerance for Air-Core Permanent Magnet Synchronous Linear Motor," IEEE Trans. Ind. Electron., vol. 68, no. 4, pp. 3336-3347, Apr. 2021

[10] S. Barmada, N. Fontana, L. Sani, D. Thomopulos, and M. Tucci, "Deep Learning and Reduced Models for Fast Optimization in Electromagnetics,' IEEE Trans. Magn., vol. 56, no. 3, pp. 1-4, Mar. 2020.

[11] J. Asanuma, S. Doi, and H. Igarashi, "Transfer Learning Through Deep Learning: Application to Topology Optimization of Electric Motor,' IEEE Trans. Magn., vol. 56, no. 3, pp. 1-4, Mar. 2020.

[12] Y. Shimizu, S. Morimoto, M. Sanada, and Y. Inoue, "Using Machine Learning to Reduce Design Time for Permanent Magnet Volume Minimization in IPMSMs for Automotive Applications," IEEJ Journal of Industry Applications, vol. 10, no. 5, pp. 554-563, 2021.

[13] S. Suzuki, S. Morimoto, M. Sanada, and Y. Inoue, "Performance comparison of IPMSMs using a low iron loss material for automotive application," in 2016 19th International Conference on Electrical Machines and Systems (ICEMS), 2016, pp. 1-6.

[14] Y. Shimizu, S. Morimoto, M. Sanada, and Y. Inoue, "Investigation of Irreversible Demagnetization Constraints in Magnet Volume Minimization Design of IPMSM for Automotive Applications Using Machine Learning," in 2021 IEEE International Electric Machines Drives Conference (IEMDC), 2021, pp. 1-6.

[15] I. Goodfellow et al., "Generative Adversarial Nets," in Advances in Neural Information Processing Systems, 2014.
[16] B. Liu, Y. Zhu, K. Song, and A. Elgammal, "Towards Faster and Stabilized GAN Training for High-fidelity Few-shot Image Synthesis," arXiv [cs.CV], 12-Jan-2021.

[17] K. He, X. Zhang, S. Ren, and J. Sun, "Deep Residual Learning for Image Recognition," arXiv [cs.CV], 10-Dec-2015.

[18] K. Deb, A. Pratap, S. Agarwal, and T. Meyarivan, "A fast and elitist multiobjective genetic algorithm: NSGA-II," IEEE Trans. Evol. Comput., vol. 6, no. 2, pp. 182-197, Apr. 2002.

[19] J. Blank and K. Deb, "Pymoo: Multi-Objective Optimization in Python," IEEE Access, vol. 8, pp. 89497-89509, 2020

[20] Y. Shimizu, "Dataset for motor parameters of IPMSM," [Online]. Available: https://ieee-dataport.org/documents/dataset-motor-parameters -ipmsm

[21] Y. Shimizu, "IPMSM-automatic-design," [Online]. Available: https://github.com/yshimizu12/IPMSM-automatic-design

Yuki Shimizu (Student Member, IEEE) received the B.E. and M.E. degrees from Osaka Prefecture University, Sakai, Japan, in 2016 and 2018, respectively. He is currently a Ph.D. student in the Department of Electrical and Information Systems, Osaka Prefecture University. His research interests include the automatic design of permanent magnet synchronous machines using machine learning. Mr. Shimizu is a student member of the IEEE. 
Shigeo Morimoto (Member, IEEE) received the B.E., M.E., and Ph.D. degrees from Osaka Prefecture University, Sakai, Japan, in 1982, 1984, and 1990, respectively. In 1984, he joined Mitsubishi Electric Corporation, Tokyo, Japan. Since 1988, he has been with the Graduate School of Engineering, Osaka Prefecture University, where he is currently a Professor. His main areas of research interest are permanent magnet synchronous machines, reluctance machines and their control systems. Dr. Morimoto is a member of the IEEE, the Society of Instrument and Control Engineers of Japan, the Institute of Systems, Control and Information Engineers, the Japan Institute of Power Electronics, and Society of Automotive Engineers of Japan.

Masayuki Sanada (Member, IEEE) received the B.E., M.E., and Ph.D. degrees from Osaka Prefecture University, Sakai, Japan, in 1989, 1991, and 1994, respectively. Since 1994, he has been with the Graduate School of Engineering, Osaka Prefecture University, where he is currently an Associate Professor. His main areas of research interest are permanent-magnet motors for direct-drive applications, their control systems, and magnetic field analysis. Dr. Sanada is a member of the IEEE, the Japan Institute of Power Electronics, and the Japan Society of Applied Electromagnetics and Mechanics.

Yukinori Inoue (Member, IEEE) received the B.E., M.E., and Ph.D. degrees from Osaka Prefecture University, Sakai, Japan, in 2005, 2007, and 2010, respectively. Since 2010, he has been with the Graduate School of Engineering, Osaka Prefecture University, where he is currently an Associate Professor. His research interests include control of electrical drives, in particular, the direct torque control of permanent magnet synchronous motors and position sensorless control of these motors. Dr. Inoue is a member of the IEEE and the Japan Institute of Power Electronics. 